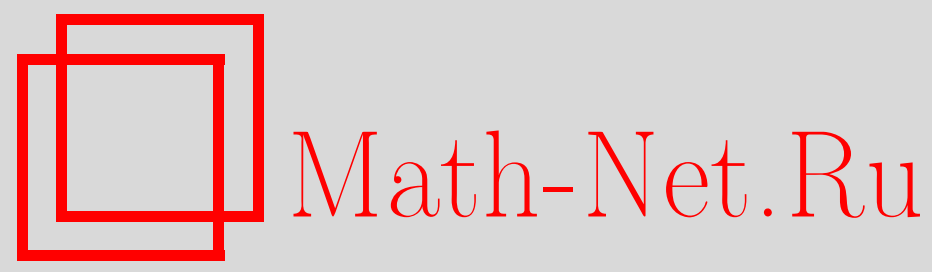

И. А. Круглов, И. В. Чередник, О существовании неотрицательных базисов в подгруппах свободных групп шрейеровых многообразий, Матем. вопр. криптогр., 2019, том 10, выпуск 4, 53-65

DOI: https://doi.org/10.4213/mvk307

Использование Общероссийского математического портала Math-Net.Ru подразумевает, что вы прочитали и согласны с пользовательским соглашением http://www . mathnet.ru/rus/agreement

Параметры загрузки:

IP : 54.84 .234 .179

26 апреля 2023 г., 13:00:03

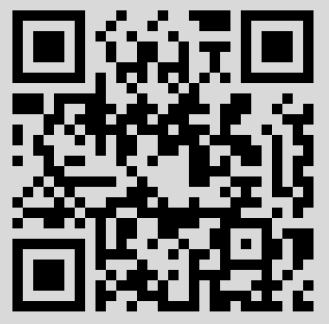


МАТЕМАТИЧЕСКИЕ ВОПРОСЫ КРИПТОГРАФИИ

2019 T. 10 № 4 C. 53-65

УДК $512.543 .1+512.543 .2$

DOI https://doi.org/10.4213/mvk307

\section{О существовании неотрицательных базисов \\ в подгруппах свободных групп шрейеровых многообразий}

И. А. Круглов ${ }^{1}$, И. В. Чередник ${ }^{2}$

${ }^{1}$ Академия криптографии Российской Федерачии, Москва

${ }^{2} O O O$ «Центр сертификачионных исследований», Москва

Получено 30.V.2016

Переработанный вариант 03.Х.2019

Аннотация. Показано, что подгруппа $H$ свободной группы $F$ с базисом $X$ обладает свободным порождающим множеством, элементы которого есть произведения положительных степеней элементов $X$ тогда и только тогда, когда $H$ порождается множеством всех своих элементов, представимых в виде произведения положительных степеней элементов $X$. Аналогичный факт доказан также для подгрупп свободных абелевых групп.

Ключевые слова: неотрицательный базис подгруппы, свободные группы, свободные абелевы группы, шрейеровы многообразия групп

On the existence of non-negative bases in subgroups of free groups of Schreier varieties

\section{A. Kruglov ${ }^{1}$, I. V. Cherednik ${ }^{2}$}

${ }^{1}$ Academy of Cryptography of the Russian Federation, Moscow

${ }^{2}$ «Certification Research Center», LLC, Moscow

Abstract. We show that a subgroup $H$ of a free group $F(X)$ has a nonnegative (with respect to $X$ ) basis if and only if $H$ is generated by the set of all its non-negative (with respect to $X$ ) elements. A similar result is proved for subgroups of free Abelian groups.

Keywords: non-negative basis of a subgroup, free groups, free Abelian groups, Schreier varieties of groups 
Пусть $G$ - произвольная группа, $\left\{g_{i}: i \in I\right\}-$ система образующих группы $G$. Элемент $g \in G$ будем называть неотрицательным nо отношению к системе образующих $\left\{g_{i}: i \in I\right\}$ группы $G$, если его можно представить в виде

$$
g=g_{i_{1}} \cdot \ldots \cdot g_{i_{t}}, i_{1}, \ldots, i_{t} \in I .
$$

Свойство быть неотрицательным по отношению к заданной системе образующих заведомо выполнено для любого элемента конечной группы, а точнее, для любого элемента периодической группы. Действительно, в этом случае любая отрицательная степень $g_{i}^{-k}$ элемента $g_{i}$, входящая в выражение $g$, равна положительной степени $g_{i}^{k\left(n_{i}-1\right)}$, где $n_{i}$ - порядок элемента $g_{i}$.

При решении практических задач возникает вопрос о построении системы образующих подгруппы $H$ группы $G$, состоящей из элементов, которые неотрицательны по отношению к заданной системе образующих $\left\{g_{i}: i \in I\right\}$ группы $G$. Здесь полезным является следующий достаточно общий результат.

Утверждение 1. Пусть $G$ - конечно-порожденная группа, $g_{1}, \ldots, g_{n}$ - система образующих группь $G$. Тогда любая подгруппа конечного индекса $H$ группы $G$ обладает конечной системой образующих, элементы которой неотрицательны по отношению $\kappa$ системе $g_{1}, \ldots, g_{n}$.

Доказательство. Сначала покажем, что подгруппа конечного индекса $H$ конечно-порожденной группы $G$ обладает конечной системой образующих элементов. Для этого рассмотрим свободную группу $F_{n}$ с базисом $x_{1}, \ldots, x_{n}$ и естественный эпиморфизм $\varphi: F_{n} \rightarrow G$, при котором $\varphi\left(x_{i}\right)=g_{i}, i \in\{1, \ldots, n\}$. Подгруппа $\varphi^{-1}(H)$ имеет конечный индекс в $F_{n}$ :

$$
\left|F_{n}: \varphi^{-1}(H)\right|=|G: H|,
$$

и согласно теореме Нильсена-Шрайера $\varphi^{-1}(H)-$ свободная группа с конечным базисом $v_{1}, \ldots, v_{t}$. Соответственно, $h_{1}=\varphi\left(v_{1}\right), \ldots, h_{t}=$ $\varphi\left(v_{t}\right)$ - конечная система образующих группы $H$.

Предположим теперь, что не существует конечной системы образующих группы $H$, все элементы которой неотрицательны по отношению к системе $g_{1}, \ldots, g_{n}$. Тогда выберем в группе $H$ такую конечную систему образующих $w_{1}, \ldots, w_{s}$, элементы которой можно выразить некоторым образом с использованием минимального в совокупности количества сомножителей $g_{1}^{-1}, \ldots, g_{n}^{-1}$. Не ограничивая общности, полагаем, что элемент $w_{1}$ не допускает неотрицательную запись в системе $g_{1}, \ldots, g_{n}$. 
Если запись элемента $w_{1}$ имеет вид $w_{1}=g_{i_{1}}^{\varepsilon_{1}} \cdot \ldots \cdot g_{i_{k-1}}^{\varepsilon_{k-1}} \cdot g_{i_{k}} \cdot g_{i_{k+1}}^{-1} \cdot \ldots \cdot g_{i_{t}}^{-1}, \quad \varepsilon_{1}, \ldots, \varepsilon_{k-1} \in\{ \pm 1\}, 0 \leqslant k<t$, то заметим, что элемент $w=g_{i_{t}} \cdot \ldots \cdot g_{i_{k+1}}$ в некоторой положительной степени $r$ ввиду конечности индекса $|G: H|$ содержится в группе $H$. Отсюда следует, что система

$$
w^{r}, w_{1} \cdot w^{r}, w_{2}, \ldots, w_{s}
$$

порождает группу $H$. Однако для ее записи в совокупности требуется строго меньше сомножителей $g_{1}^{-1}, \ldots, g_{n}^{-1}$, чем для системы образующих элементов $w_{1}, \ldots, w_{s}$. Получено противоречие.

Если же запись элемента $w_{1}$ имеет вид

$$
\begin{aligned}
w_{1}=g_{i_{1}}^{\varepsilon_{1}} \cdot \ldots \cdot g_{i_{l-1}}^{\varepsilon_{l-1}} \cdot g_{i_{l}} \cdot g_{i_{l+1}}^{-1} \cdot \ldots g_{i_{k}}^{-1} \cdot g_{i_{k+1}} \cdot \ldots \cdot g_{i_{t}}, \\
\varepsilon_{1}, \ldots, \varepsilon_{l-1} \in\{ \pm 1\}, 0 \leqslant l<k<t,
\end{aligned}
$$

то ввиду конечности индекса $|G: H|$ элемент $w=g_{i_{k+1}} \cdot \ldots \cdot g_{i_{t}}$ в некоторой положительной степени $r$ содержится в группе $H$ и элемент

$$
v=\left(g_{i_{k+1}} \cdot \ldots \cdot g_{i_{t}}\right)^{r-1} \cdot g_{i_{k}} \cdot \ldots \cdot g_{i_{l+1}}
$$

в некоторой конечной степени $m$ содержится в группе $H$. В таком случае система

$$
v^{m}, w^{r}, w_{1} \cdot w^{-r} \cdot v^{m}, w_{2}, \ldots, w_{s}
$$

порождает группу $H$, и для ее записи в совокупности требуется строго меньше сомножителей $g_{1}^{-1}, \ldots, g_{n}^{-1}$, чем для системы образующих элементов $w_{1}, \ldots, w_{s}$. Снова получено противоречие.

В целом для случая конечно-порожденных групп утверждение 1 неулучшаемо. С одной стороны, пример

$$
G=\mathbb{Z}^{2},\left\{g_{1}, g_{2}\right\}=\{(1,0),(0,1)\}, H=\langle(1,-1)\rangle
$$

наглядно показывает, что нельзя отказаться от условия конечности индекса $|G: H|$. С другой стороны, в случае произвольной группы $G$ отсутствуют какие-то общие результаты о мощности систем образующих подгруппы $H$ даже в случае ее конечного индекса.

Однако для практически важной задачи о существовании неотрицательных базисов подгрупп свободных групп можно получить содержательные результаты. Для конечно-порожденных подгрупп свободных групп конечного ранга в работе [7] была доказана следующая теорема. 
Теорема 1 ([7], следствие 3.6). Пусть $F_{n}-$ конечно-порожденная свободная группа с базисом $x_{1}, \ldots, x_{n}$. Конечно-порожденная подгруппа $H$ в группе $F_{n}$ обладает неотрииательным по отношению $к$ системе $x_{1}, \ldots, x_{n}$ базисом в том и только в том случае, когда Н порождается множеством всех своих элементов, которые неотрицательны по отношению $к$ системе $x_{1}, \ldots, x_{n}$.

Следствие 1. Пусть $F_{n}$ - конечно-порожденная свободная группа с базисом $x_{1}, \ldots, x_{n}$. Тогда любая подгруппа конечного индекса $H$ в группе $F_{n}$ обладает неотрицательным базисом по отношению $к$ сиcmeме $x_{1}, \ldots, x_{n}$.

Следствие 1 несложно вывести из теоремы 1 и утверждения 1.

Заметим также, что последний результат, в совокупности с известной формулой Нильсена-Шрейера, позволяет уточнить доказанное выше утверждение 1 следующим образом.

Следствие 2. Пусть $G$ - конечно-порожденная группа с множеством образующих элементов $g_{1}, \ldots, g_{n}$. Тогда любая подгруппа конечного индекса $H$ в группе $G$ обладает неотрицательной по отношению $\kappa$ системе $g_{1}, \ldots, g_{n}$ системой образуюших $h_{1}, \ldots, h_{t}$ с числом элеменmов $t \leqslant|G: H| \cdot(n-1)+1$.

Еще один класс групп, важных с практической точки зрения, представляют свободные абелевы группы конечного ранга. Пусть $\left(A_{n},+\right)-$ свободная абелева группа конечного ранга $n \geqslant 1$ с базисом $\alpha_{1}, \ldots, \alpha_{n}$. Любая подгруппа конечного индекса в группе $A_{n}$ является свободной абелевой группой конечного ранга $n$. В [9] А. А. Нечаевым была предложена задача описать все подгруппы конечного индекса в группе $A_{n}$, которые порождаются множеством всех своих элементов, неотрицательных по отношению к системе $\alpha_{1}, \ldots, \alpha_{n}$. В [6] И. В. Чередник получил следующий результат.

Теорема 2 ([6], следствие 2). Пусть $A_{n}-$ конечно-порожденная свободная абелева группа с базисом $\alpha_{1}, \ldots, \alpha_{n}$. Подгруппа $B$ группь $A_{n}$ обладает неотрицательным по отношению $\kappa$ системе $\alpha_{1}, \ldots, \alpha_{n}$ базисом в том и только в том случае, когда $B$ порождается множеством всех своих элементов, неотрицательных по отношению $к$ системе $\alpha_{1}, \ldots, \alpha_{n}$.

Отметим, что теорема 2 не является непосредственным следствием из теоремы 1 , что объясняется разной природой свободных и свободных абелевых групп. 
Следствие 3. Любая подгруппа $B$ конечного индекса свободной абелевой группы $A_{n}$ обладает базисом $\beta_{1}, \ldots, \beta_{n}$, элементы которого неотрищательны по отношению $к$ системе образуюших $\alpha_{1}, \ldots, \alpha_{n}$.

Следствие 3 вытекает из теоремы 2 с учетом утверждения 1.

На наш взгляд, приведенные выше теоремы 1, 2 и следствия 1, 3 весьма сложно обобщить непосредственно на случай свободных групп иных многообразий. Это объясняется тем, что свободные группы только трех многообразий: все группы, абелевы группы, абелевы группы (фиксированной) простой экспоненты, - обладают тем свойством, что любая их подгруппа является относительно свободной группой того же многообразия и, соответственно, имеет базисы, мощность которых инвариантна (такие многообразия называют шрейеровыми, см. [10], [5] (стр. 154), [2] (стр. 472)).

Остается заметить, что понятие неотрицательного элемента в случае периодических групп является вырожденным и, как следствие, любая подгруппа свободной группы многообразия абелевых групп простой экспоненты обладает неотрицательным базисом.

В настоящей работе доказываются обобщение теоремы 2 на случай произвольной подгруппы свободной абелевой группы $A$ произвольного ранга, а также обобщение теоремы 1 на случай произвольной подгруппы свободной группы $F$ произвольного ранга.

Начнем с обобщения теоремы 2. Напомним, что свободная абелева группа $A$ с базисом $\mathcal{A}=\left\{\alpha_{s}: s \in S\right\}$ есть прямая сумма $\sum_{s \in S}\left\langle\alpha_{s}\right\rangle$ бесконечных циклических групп $\left\langle\alpha_{s}\right\rangle$ (см., например, [2], стр. 118) и каждый элемент группы $A$ однозначно представляется в виде конечной суммы

$$
c_{1} \alpha_{s_{1}}+\ldots+c_{n} \alpha_{s_{n}}
$$

целочисленно кратных попарно различных элементов $\alpha_{s_{1}}, \ldots, \alpha_{s_{n}} \in \mathcal{A}$. Теорема 3. Пусть $(A,+)$ - свободная абелева группа с базисом $\mathcal{A}=\left\{\alpha_{s}: s \in S\right\}$. Подгруппа $B$ группь $A$ обладает неотрицательным по отношению $\kappa \mathcal{A}$ базисом в том и только в том случае, когда $B$ порождается множеством всех своих элементов, неотрицательных по отношению $\kappa \mathcal{A}$.

Доказательство. Необходимость очевидна, докажем достаточность условия.

Пусть $\mathcal{C}=\left\{\gamma_{k}: k \in K\right\}-$ некоторая система образующих группы $B$, состоящая из неотрицательных по отношению к базису $\mathcal{A}$ элементов. 
Можно считать, что система $\mathcal{C}$ вполне упорядочена и не содержит элементов, целочисленно кратных элементам группы $B$. Доказательство существования неотрицательного базиса группы $B$ проведем стандартным образом с использованием трансфинитной индукции, а именно: предложим индуктивный процесс построения линейно независимой системы $\left\{\gamma_{i}^{*}: i \in I\right\}$ неотрицательных элементов, через которые линейно выражается каждый элемент системы $\mathcal{C}$.

Выберем некоторый базис $\left\{\beta_{j}: j \in J_{1}\right\}$ группы $B$. Поскольку $\gamma_{1} \in C$ не является целочисленно кратным элемента группы $B$, то коэффициенты из его разложения

$$
\gamma_{1}=c_{1} \beta_{j_{1}}+\ldots+c_{t} \beta_{j_{t}}, j_{1}, \ldots, j_{t} \in J_{1},
$$

взаимно просты в совокупности, т. е. НОД $\left(c_{1}, \ldots, c_{t}\right)=1$. Как известно из теории свободных абелевых групп конечного ранга, элемент $\gamma_{1}$ можно дополнить до базиса $\gamma_{1}, \gamma_{2}^{\prime}, \ldots, \gamma_{t}^{\prime}$ свободной абелевой группы, порожденной системой $\beta_{j_{1}}, \ldots, \beta_{j_{t}}$. Пользуясь этим, элемент $\gamma_{1}$ дополним до базиса группы $B$ :

$$
\begin{aligned}
\left\{\gamma_{i}^{*}: i \in I_{2}\right\} \cup\left\{\beta_{j}: j \in J_{2}\right\} & \\
& =\left\{\gamma_{1}\right\} \cup\left(\left(\left\{\beta_{j}: j \in J_{1}\right\} \backslash\left\{\beta_{j_{1}}, \ldots, \beta_{j_{t}}\right\}\right) \cup\left\{\gamma_{2}^{\prime}, \ldots, \gamma_{t}^{\prime}\right\}\right) .
\end{aligned}
$$

Предположим теперь, что построен такой базис

$$
\left\{\gamma_{i}^{*}: i \in I_{k}\right\} \cup\left\{\beta_{j}: j \in J_{k}\right\}
$$

группы $B$, что $\left\{\gamma_{i}^{*}: i \in I_{k}\right\}$ - линейно независимая система неотрицательных по отношению к $\mathcal{A}$ элементов, через которую линейно выражается любой элемент $\gamma_{s}, s<k$.

Если $\gamma_{k}$ линейно выражается через $\left\{\gamma_{i}^{*}: i \in I_{k}\right\}$, то положим

$$
\left\{\gamma_{i}^{*}: i \in I_{k+1}\right\}=\left\{\gamma_{i}^{*}: i \in I_{k}\right\}, \quad\left\{\beta_{j}: j \in J_{k+1}\right\}=\left\{\beta_{j}: j \in J_{k}\right\} .
$$

Если же $\gamma_{k}$ не выражается линейно через $\left\{\gamma_{i}^{*}: i \in I_{k}\right\}$, то его разложение в базисе $\left\{\gamma_{i}^{*}: i \in I_{k}\right\} \cup\left\{\beta_{j}: j \in J_{k}\right\}$ имеет вид

$$
\gamma_{k}=c_{1} \gamma_{i_{1}}^{*}+\ldots+c_{s} \gamma_{i_{s}}^{*}+d_{1} \beta_{j_{1}}+\ldots+d_{t} \beta_{j_{t}},\left(d_{1}, \ldots, d_{t}\right) \neq(0, \ldots, 0) .
$$

Пусть $\left(d_{1}, \ldots, d_{t}\right)=d$. Выберем неотрицательные целые числа $r_{1}, \ldots, r_{s}$ таким образом, что $d \mid\left(c_{i}+r_{i}\right), i \in\{1, \ldots, s\}$, и положим

$$
\begin{aligned}
\gamma_{k}^{\prime} & =\gamma_{k}+r_{1} \gamma_{i_{1}}^{*}+\ldots+r_{s} \gamma_{i_{s}}^{*} \\
& =\left(c_{1}+r_{1}\right) \gamma_{i_{1}}^{*}+\ldots+\left(c_{s}+r_{s}\right) \gamma_{i_{s}}^{*}+d_{1} \beta_{j_{1}}+\ldots+d_{t} \beta_{j_{t}} .
\end{aligned}
$$


Ввиду того, что элементы $\gamma_{i_{1}}^{*}, \ldots, \gamma_{i_{s}}^{*}, \gamma_{k}$ неотрицательны по отношению к $\mathcal{A}$ и $r_{1}, \ldots, r_{s} \geqslant 0$, элемент $\gamma_{k}^{\prime}$ также является неотрицательным по отношению к $\mathcal{A}$. Остается заметить, что элемент

$$
\gamma^{*}=\frac{c_{1}+r_{1}}{d} \gamma_{i_{1}}^{*}+\ldots+\frac{c_{s}+r_{s}}{d} \gamma_{i_{s}}^{*}+\frac{d_{1}}{d} \beta_{j_{1}}+\ldots+\frac{d_{t}}{d} \beta_{j_{t}}
$$

неотрицателен по отношению к $\mathcal{A}$, а система $\gamma_{i_{1}}^{*}, \ldots, \gamma_{i_{s}}^{*}, \gamma^{*}$ дополнима до некоторого базиса $\gamma_{i_{1}}^{*}, \ldots, \gamma_{i_{s}}^{*}, \gamma^{*}, \gamma_{2}^{\prime}, \ldots, \gamma_{t}^{\prime}$ свободной абелевой группы, порожденной системой $\gamma_{i_{1}}^{*}, \ldots, \gamma_{i_{s}}^{*}, \beta_{j_{1}}, \ldots, \beta_{j_{t}}$. Итак, мы построили базис

$$
\begin{aligned}
& \left\{\gamma_{i}^{*}: i \in I_{k+1}\right\} \cup\left\{\beta_{j}: j \in J_{k+1}\right\} \\
=\left(\left\{\gamma_{i}^{*}:\right.\right. & \left.\left.i \in I_{k}\right\} \cup\left\{\gamma^{*}\right\}\right) \cup\left(\left(\left\{\beta_{j}: j \in J_{k}\right\} \backslash\left\{\beta_{j_{1}}, \ldots, \beta_{j_{t}}\right\}\right) \cup\left\{\gamma_{2}^{\prime}, \ldots, \gamma_{t}^{\prime}\right\}\right)
\end{aligned}
$$

группы $B$ с тем свойством, что $\left\{\gamma_{i}^{*}: i \in I_{k+1}\right\}-$ линейно независимая система неотрицательных по отношению к базису $\mathcal{A}$ элементов, через которую линейно выражается любой элемент $\gamma_{s}, s<k+1$.

Таким образом, доказано существование неубывающей цепочки систем

$$
\left\{\gamma_{i}^{*}: i \in I_{k}\right\}, k \in K,
$$

с обозначенными выше свойствами. Теперь легко видеть, что система

$$
\bigcup_{k \in K}\left\{\gamma_{i}^{*}: i \in I_{k}\right\}
$$

является базисом группы $B$, состоящим из неотрицательных по отношению к базису $\mathcal{A}$ элементов.

Перейдем к обобщению теоремы 1 . В наших рассуждениях будем использовать представление свободных групп в виде фундаментальных групп связных графов. Приведем необходимые сведения (см. $\S 2,3$ главы 3 книги [3], а также $\S 3$ главы 2 книги [1]).

Графом Г называется совокупность $(V, E, \alpha, \omega, \eta)$, состоящая из непустого множества $V$ (множество вершин), множества $E$ (множество ребер), отображений $\alpha: E \rightarrow V, \omega: E \rightarrow V$, а также преобразования $\eta: E \rightarrow E$, для которых при любом $e \in E$ выполнены равенства

$$
\eta(e) \neq e, \quad \eta^{2}(e)=e, \quad \alpha(\eta(e))=\omega(e), \quad \omega(\eta(e))=\alpha(e) .
$$

Для любого $e \in E$ вершина $\alpha(e)$ называется началом, а вершина $\omega(e)$ - кониом ребра $е$. Ребра $е$ и $\eta(e)$ называются противоположными, для любого ребра е пара $\{e, \eta(e)\}$ называется неориентированным ребром. 
Отметим, что отображение $e \mapsto(\alpha(e), \omega(e))$ не обязательно предполагается инъективным, т. е. различные ребра могут иметь одинаковые начало и конец. В конечном графе с множеством ребер $E$ число неориентированных ребер равно $\frac{|E|}{2}$.

Путем z в графе Г называется конечная последовательность ребер

$$
z=e_{i_{1}}, \ldots, e_{i_{k}}, \quad k \geqslant 1
$$

в которой для каждого $s=1,2, \ldots, k-1$ выполнено равенство $\alpha\left(e_{i_{s+1}}\right)=\omega\left(e_{i_{s}}\right)$. При этом вершина $\alpha(z)=\alpha\left(e_{i_{1}}\right)$ называется началом, вершина $\omega(z)=\omega\left(e_{j_{k}}\right)-$ конц,м пути $z$. Путь

$$
\eta\left(e_{i_{k}}\right), \ldots, \eta\left(e_{i_{1}}\right)
$$

будем называть обратным к пути $z=e_{i_{1}}, \ldots, e_{i_{k}}$ и обозначать через $z^{-1}$.

Граф Г называется связным, если для любых двух различных вершин $v_{1}, v_{2} \in V$ существует путь в $\Gamma$ с началом $v_{1}$ и концом $v_{2}$. Далее мы рассматриваем только связные графы с непустым множеством ребер.

Если для вершины $v \in V$ и пути $z$ выполнены равенства $\alpha(z)=$ $\omega(z)=v$, то путь $z$ называется петлей с кониом $v$. Кроме того, каждой вершине $v \in V$ ставится в соответствие пустая петля $1_{v}$ с концом $v$, не содержащая ребер.

Обозначим через $Z_{v}$ множество всех петель в графе $\Gamma$ с фиксированным концом $v$. Для любых петель $z, w \in Z_{v}$ последовательность $(z, w)$, полученная приписыванием $w$ справа от $z$, является петлей из множества $Z_{v}$. Это определяет полугрупповую операцию на множестве $Z_{v}$.

Две петли $z_{1}, z_{2} \in Z_{v}$ назовем эквивалентными, если $z_{2}$ можно получить из $z_{1}$ конечным числом вставок и вычеркиваний подпутей вида $e, \eta(e)$. Введенное отношение действительно является отношением эквивалентности на множестве $Z_{v}$, и оно согласовано с указанной выше полугрупповой операцией. Фактор-полугруппу $\Phi(\Gamma, v)$ полугруппы $Z_{v}$ по данному отношению эквивалентности называют фундаментальной группой графа $\Gamma$ в точке $v$. В дальнейшем для любой петли $z \in Z_{v}$ класс эквивалентности с представителем $z$ будем обозначать через $\bar{z}$.

Хорошо известно, что $\Phi(\Gamma, v)$ является свободной группой, и если $\Gamma-$ конечный граф, то $\Phi(\Gamma, v)-$ свободная группа ранга $\frac{|E|}{2}-|V|+1$ (см., например, предложения 2.1 и 2.2 в книге [3]). Также известно (см., например, $\S 2$ главы 3 книги [3]), что структура $\Phi(\Gamma, v)$ не зависит от выбора вершины $v$ : группы $\Phi\left(\Gamma, v_{1}\right)$ и $\Phi\left(\Gamma, v_{2}\right)$ сопряжены и, следовательно, изоморфны. 
Путь $z$ в графе Г называется приведенным, если он не содержит подпути вида $(e, \eta(e))$. Можно показать, что каждый класс $\bar{z} \in \Phi(\Gamma, v)$ содержит единственную приведенную петлю.

Вершину $v^{\prime} \in V$ будем называть сильно связанной с вершиной $v$, если существует такая приведенная петля

$$
z=e_{i_{1}}, \ldots, e_{i_{k}} \in Z_{v}
$$

что $v^{\prime}=\alpha\left(e_{i_{s}}\right)$ для некоторого $s \in 1, \ldots, k$. Обозначим множество всех вершин графа $\Gamma$, сильно связанных с $v$, через $V_{v}$ и определим подграф

$$
\Gamma_{v}=\left(V_{v}, E_{v}\right), E_{v}=\left\{e \in E: \alpha(e), \omega(e) \in V_{v}\right\} .
$$

Вообще, группа $\Phi\left(\Gamma_{v}, v\right)$ очевидным образом изоморфна группе $\Phi(\Gamma, v)$. Однако для удобства изложения бывает предпочтительнее рассматривать граф $\Gamma_{v}$ и группу $\Phi\left(\Gamma_{v}, v\right)$.

Определим понятие сильной связности графа, следуя [3]. Выбрав произвольно по одному ребру из каждого неориентированного ребра графа $\Gamma$, получим некоторое подмножество $E_{0} \subset E$. Будем говорить, что подмножество $E_{0}$ определяет ориентацию ребер в графе Г. В случае конечного графа $\Gamma$ выполнено равенство $\left|E_{0}\right|=\frac{|E|}{2}$, т. е. имеется $2^{\frac{|E|}{2}}$ способов определить ориентацию ребер в графе $\Gamma$.

Граф Г называется сильно связным относительно ориентации ребер, определяемой подмножеством $E_{0}$, если для любых двух вершин $v_{1}, v_{2} \in V$ существует такой путь $z$ в $\Gamma$ с началом $v_{1}$ и концом $v_{2}$, что все ребра пути $z$ содержатся в множестве $E_{0}$. Отметим, что для любой вершины $v$ произвольная петля $z \in Z_{v}$, все ребра которой содержатся в множестве $E_{0}$, является приведенной, а потому для сильно связного относительно некоторой ориентации $E_{0}$ графа $\Gamma$ выполнено равенство $\Gamma_{v}=\Gamma$.

В 1973 году Ю. Н. Горчинский, в связи с исследованием специальных алгоритмов обработки информации, опубликовал в ведомственном научном журнале утверждение, эквивалентное приведенному выше следствию 1. При этом наиболее содержательная часть доказательства была оформлена в виде следующего отдельного результата.

Лемма 1 (Ю.Н. Горчинский, 1973). Пусть конечный граф Г с множеством вершин $V$ и множеством ребер Е является сильно связным относительно ориентации ребер, определяемой некоторым подмножеством $E_{0} \subset E$. Тогда для любой вершины $v \in V$ существует такая система петель $z_{1}, \ldots, z_{t} \in Z_{v}$, что при любом $i=1, \ldots, t$ все 
ребра петли $z_{i}$ содержатся в множестве $E_{0}$ и множество соответствующих классов эквивалентности $\bar{z}_{1}, \ldots, \bar{z}_{t}$ является базисом груп$n ъ \iota(\Gamma, v)$.

В данной работе мы предлагаем доказательство обобщения леммы 1 на случай произвольного сильно связного графа, проведенное с использованием более современных методов $([3,4,8])$.

Лемма 2. Пусть граф Г с множеством вершин $V$ и множеством ребер Е является сильно связным относительно ориентачии ребер, определяемой некоторым подмножеством $E_{0} \subset E$. Тогда для любой вериины $v \in V$ существует такая система петель $\left\{z_{i}: i \in I\right\}$, ито при любом $i \in I$ все ребра петли $z_{i}$ содержатся в множестве $E_{0} u$ множество соответствующих классов эквивалентности $\left\{\bar{z}_{i}: i \in I\right\}$ является базисом группы $\Phi(\Gamma, v)$.

Доказательство. Множество всех корневых деревьев сильно связного графа $\Gamma_{0}=\left(V, E_{0}\right)$, вырастающих из фиксированной вершины $v \in V$, является частично упорядоченным и обладает верхней гранью. Согласно лемме Цорна в данном множестве можно выбрать некоторый максимальный элемент $S=\left(V_{S}, E_{S}\right)$, который является остовным деревом для графа $\Gamma_{0}$, т. е. $V_{S}=V$ (см., например, $\S 2.2$ книги [8]). При этом для каждой вершины $v^{\prime} \in V$ в дереве $S$ существует единственный путь $z_{v, v^{\prime}}^{S}$ с началом $v$ и концом $v^{\prime}$.

Пусть $E_{0} \backslash E_{S}=\left\{e_{i}: i \in I\right\}$. Тогда, как известно (см., например, предложения 2.1 и 2.2 в книге [3]), система петель

$$
w_{i}=z_{v, \alpha\left(e_{i}\right)}^{S}, e_{i},\left(z_{v, \omega\left(e_{i}\right)}^{S}\right)^{-1}, i \in I,
$$

обладает тем свойством, что $\left\{\bar{w}_{i}: i \in I\right\}-$ базис группы $\Phi(\Gamma, v)$.

Выберем дополнительно в сильно связном графе $\Gamma_{0}=\left(V, E_{0}\right)$ произвольное остовное корневое дерево $T=\left(V, E_{T}\right)$, врастающее в вершину $v$. Для каждой вершины $v^{\prime} \in V$ в дереве $T$ существует единственный путь $z_{v^{\prime}, v}^{T}$ с началом $v^{\prime}$ и концом $v$.

Рассмотрим систему петель

$$
z_{i}=z_{v, \alpha\left(e_{i}\right)}^{S}, e_{i}, z_{\omega\left(e_{i}\right), v}^{T}, i \in I,
$$

в которой каждая петля по построению содержит только ребра из $E_{0}$. Покажем, что система $\left\{\bar{z}_{i}: i \in I\right\}$ порождает базис $\left\{\bar{w}_{i}: i \in I\right\}$. Действительно, если

$z_{\omega\left(e_{i}\right), v}^{T}=e_{j_{1}}, \ldots, e_{j_{r-1}}, e_{j_{r}}, e_{j_{r+1}}, \ldots, e_{j_{l}}, \quad e_{j_{1}}, \ldots, e_{j_{r-1}} \in E_{S}, e_{j_{r}} \in E_{T} \backslash E_{S}$, 
то ввиду единственности пути в $S$ с началом $v$ и концом $\alpha\left(e_{j_{r}}\right)$ выполнено равенство

$$
z_{v, \alpha\left(e_{j_{r}}\right)}^{S}=z_{v, \omega\left(e_{i}\right)}^{S}, e_{j_{1}}, \ldots, e_{j_{r-1}},
$$

а ввиду единственности пути в $T$ с началом $\omega\left(e_{j_{r}}\right)$ и концом $v$ - равенство

$$
z_{\omega\left(e_{j_{r}}\right), v}^{T}=e_{j_{r+1}}, \ldots, e_{j_{l}}
$$

Остается заметить, что

$$
\begin{gathered}
\bar{z}_{i} \cdot\left(\bar{z}_{j_{r}}\right)^{-1}=\overline{z_{i},\left(z_{j_{r}}\right)^{-1}}=\overline{z_{v, \alpha\left(e_{i}\right)}^{S}, e_{i}, z_{\omega\left(e_{i}\right), v}^{T},\left(z_{v, \alpha\left(e_{j_{r}}\right)}^{S}, e_{j_{r}}, z_{\omega\left(e_{j_{r}}\right), v}^{T}\right)^{-1}} \\
=\overline{z_{v, \alpha\left(e_{i}\right)}^{S}, e_{i}, e_{j_{1}}, \ldots, e_{j_{r-1}}, e_{j_{r}}, e_{j_{r+1}}, \ldots, e_{j_{l}},\left(z_{v, \alpha\left(e_{j_{r}}\right)}^{S}, e_{j_{r}}, z_{\omega\left(e_{j_{r}}\right), v}^{T}\right)^{-1}} \\
=\overline{z_{v, \alpha\left(e_{i}\right)}^{S}, e_{i}, e_{j_{1}}, \ldots, e_{j_{r-1}}, e_{j_{r}}, z_{\omega\left(e_{j_{r}}\right), v}^{T},\left(z_{\omega\left(e_{j_{r}}\right), v}^{T}\right)^{-1}, \eta\left(e_{j_{r}}\right),\left(z_{v, \alpha\left(e_{j_{r}}\right)}^{S}\right)^{-1}} \\
=\overline{z_{v, \alpha\left(e_{i}\right)}^{S}, e_{i}, e_{j_{1}}, \ldots, e_{j_{r-1}}, \eta\left(e_{j_{r-1}}\right), \ldots, \eta\left(e_{j_{1}}\right),\left(z_{v, \omega\left(e_{i}\right)}^{S}\right)^{-1}} \\
=\overline{z_{v, \alpha\left(e_{i}\right)}^{S}, e_{i},\left(z_{v, \omega\left(e_{i}\right)}^{S}\right)^{-1}}=\bar{w}_{i} .
\end{gathered}
$$

Таким образом, $\left\{\bar{z}_{i}: i \in I\right\}$ - система образующих свободной группы $\Phi(\Gamma, v)$. Покажем, что $\left\{\bar{z}_{i}: i \in I\right\}$ - свободная система образующих этой группы.

Рассмотрим произвольную конечную систему петель

$$
z_{i_{1}}=z_{v, \alpha\left(e_{i_{1}}\right)}^{S}, e_{i_{1}}, z_{\omega\left(e_{i_{1}}\right), v}^{T}, \ldots, z_{i_{k}}=z_{v, \alpha\left(e_{i_{k}}\right)}^{S}, e_{i_{k}}, z_{\omega\left(e_{i_{k}}\right), v}^{T} .
$$

Пусть $e_{i_{k+1}}, \ldots, e_{i_{n}}$ - все ребра из множества $E_{T} \backslash E_{S}$, содержащиеся в путях $z_{\omega\left(e_{i_{1}}\right), v}^{T}, \ldots, z_{\omega\left(e_{i_{k}}\right), v}^{T}$. Тогда системы петель

$$
w_{i_{1}}, \ldots, w_{i_{k}}, w_{i_{k+1}}, \ldots, w_{i_{n}}
$$

И

$$
z_{i_{1}}, \ldots, z_{i_{k}}, z_{i_{k+1}}, \ldots, z_{i_{n}}
$$

обладают тем свойством, что $\bar{w}_{i_{1}}, \ldots, \bar{w}_{i_{n}}$ порождает $\bar{z}_{i_{1}}, \ldots, \bar{z}_{i_{n}}$, и наоборот. Таким образом, система $\bar{z}_{i_{1}}, \ldots, \bar{z}_{i_{n}}$ является системой образующих конечно-порожденной свободной группы с базисом $\bar{w}_{i_{1}}, \ldots, \bar{w}_{i_{n}}$, а значит (см. предложение 2.7 в книге [3] или следствие 2.13.1 в книге [4]), $\bar{z}_{i_{1}}, \ldots, \bar{z}_{i_{n}}-$ свободная система образующих. Итак, мы показали, что произвольная конечная система образующих $\bar{z}_{i_{1}}, \ldots, \bar{z}_{i_{k}}$ свободна.

Доказанное обобщение леммы 1 позволяет обосновать основной результат настоящей работы. 
Теорема 4. Пусть $F-$ свободная группа с базисом $X$. Подгруппа $H$ группь $F$ обладает неотрицательным по отношению $к X$ базисом в том и только в том случае, когда $H$ порождается множеством всех своих элементов, которые неотрицательны по отношению $\kappa X$.

Доказательство. Необходимость прямо следует из определения; докажем достаточность условия.

Рассмотрим граф $Г$ с множеством вершин $V=\{H g: g \in G\}$ и множеством ребер

$$
E=\left\{(H g, H g s): H g \in V, s \in X \cup X^{-1}\right\} .
$$

Элемент $s$ будем называть меткой ребра ( $\mathrm{Hg}, \mathrm{Hgs})$. Если $e_{1}, \ldots, e_{t}-$ путь в графе $\Gamma$ и ребра $e_{1}, \ldots, e_{t}$ имеют метки $s_{1}, \ldots, s_{t}$ соответственно, то элемент $s_{1} \cdot \ldots \cdot s_{t} \in G$ будем называть меткой nути $e_{1}, \ldots, e_{t}$. Подгруппа $H$ состоит из меток всех петель в графе $Г$ с концом в вершине $H$, при этом отображение

$$
\overline{e_{1}, \ldots, e_{t}} \mapsto s_{1} \cdot \ldots \cdot s_{t}
$$

определенное на множестве классов петель с концом $H$, является изоморфизмом групп $\Phi(\Gamma, H)$ и $H$.

Далее для удобства вместо графа $Г$ и группы $\Phi(\Gamma, H)$ будем рассматривать определенный в соотношении (1) граф $\Gamma_{H}($ при $v=H$ ) и соответствующую группу $\Phi\left(\Gamma_{H}, H\right)$. Обозначим через $\Gamma_{0}$ подграф $\Gamma_{H}$ с множеством вершин $V_{H}$ и множеством ребер

$$
E_{0}=\left\{(H g, H g s) \in E_{H}: s \in X\right\} .
$$

Поскольку группа $H$ порождается множеством всех своих неотрицательных элементов, то группа $\Phi\left(\Gamma_{H}, H\right)$ обладает системой образующих элементов, приведенные представители которых содержатся в $\Gamma_{0}$.

Методом «от противного» можно показать, что для любой вершины $H g \in V_{H}$ в графе $\Gamma_{0}$ существует хотя бы одна петля с концом $H$, содержащая данную вершину. Последнее означает, что граф $\Gamma_{H}$ сильно связен относительно ориентации $E_{0}$ и, следовательно, к графу $\Gamma_{H}$ можно применить лемму 2.

Таким образом, группа $\Phi\left(\Gamma_{H}, H\right)$ обладает свободной системой образующих элементов, приведенные представители которых содержатся в $\Gamma_{0}$, а соответствующие им метки образуют неотрицательный относительно $X$ базис группы $H$. 


\section{Список литературы}

[1] Бахтурин Ю.А., Основные структуры современной алгебры, М.: Наука. Гл. ред. физ.мат. лит., 1990, 320 с.

[2] Курош А. Г., Теория групn, М.: Наука, 1967, 648 с.

[3] Линдон Р., Шупп П., Комбинаторная теория групn, М.: Мир, 1980, 447 с.

[4] Магнус В., Каррас, А., Солитэр Д., Комбинаторная теория групn, М.: Наука, 1974, $456 \mathrm{c.}$

[5] Нейманн Х., Многообразия групn, М.: Мир, 1969, 264 с.

[6] Чередник И.В., "Неотрицательный базис решетки”, Дискретная математика, 26:3 (2014), 127-135.

[7] Khan B., "Positively generated subgroups of free groups and the Hanna Neumann conjecture", Contemporary Mathematics, 296, eds. R. Gilman et al., AMS, 2002.

[8] Horizons of Combinatorics, eds. Gyori E., Katona G. O. H., Lovasz L., Springer Science \& Business Media, 2008, 280 pp.

[9] Kurakin V.L., Kuzmin A.S., Mikhalev A.V., Nechaev A.A., "Linear recurring sequences over rings and modules", J. Math. Sci., 76:6 (1995), 2793-2915.

[10] Neumann P.M. , Wiegold J., "Schreier varieties of groups", Matematika, 12:4 (1968), 42-49. 